\title{
The kinetic and biological activity of different loaded rhBMP-2 calcium phosphate cement implants in rats
}

\author{
Esther W. H. Bodde, ${ }^{1}$ Otto C. Boerman, ${ }^{2}$ Frans G. M. Russel, ${ }^{3}$ Antonios G. Mikos, ${ }^{4}$ \\ Paul H. M. Spauwen, ${ }^{5}$ John A. Jansen ${ }^{1}$ \\ ${ }^{1}$ Department of Periodontology and Biomaterials, Radboud University Nijmegen Medical Center, \\ Nijmegen, The Netherlands \\ ${ }^{2}$ Department of Nuclear Medicine, Radboud University Nijmegen Medical Center, Nijmegen, The Netherlands \\ ${ }^{3}$ Department of Pharmacology and Toxicology, Radboud University Nijmegen Medical Center, Nijmegen, \\ The Netherlands \\ ${ }^{4}$ Department of Bioengineering, Rice University, Houston, Texas \\ ${ }^{5}$ Department of Plastic and Reconstructive Surgery, Radboud University Nijmegen Medical Center, Nijmegen, \\ The Netherlands
}

Received 2 June 2007; revised 17 September 2007; accepted 18 October 2007

Published online 15 January 2008 in Wiley InterScience (www.interscience.wiley.com). DOI: 10.1002/jbm.a.31830

\begin{abstract}
The healing of large bone defects can be improved by osteogenic bone graft substitutes, due to growth factor inclusion. A sustained release of these growth factors provides more efficient bioactivity when compared with burst release and might reduce the dose required for bone regeneration, which is desirable for socioeconomical and safety reasons. In this study, we compared different rhBMP-2 loadings in a sustained release system of $\mathrm{CaP}$ cement and PLGA-microparticles and were able to couple kinetic to biological activity data. Fifty-two rats received a critical-size cranial defect, which was left open or filled with the cement composites. The implants consisted of plain, high, and five-fold lower dose rhBMP-2 groups. Implantation time was 4 and 12 weeks. Longitudinal in vivo release was monitored by scintigraphic imaging of ${ }^{131}$ I-labeled rhBMP-2. Quantitative analysis of the scintigraphic images revealed a sustained
\end{abstract}

release of ${ }^{131}$ I-rhBMP-2 for both doses, with different release profiles between the two loadings. However, around $70 \%$ of the initial dose was retained in both implant formulations. Although low amounts of rhBMP-2 were released $(2.4 \pm 0.8 \mu \mathrm{g}$ in 5 weeks), histology showed defect bridging in the high-dose implants. Release out of the low-dose implants was not sufficient to enhance bone formation. Implant degradation was limited in all formulations, but was mainly seen in the high-dose group. Low amounts of sustained released rhBMP-2 were sufficient to bridge critically sized defects. A substantial amount of rhBMP-2 was retained in the implants because of the slow release rate and the limited degradation. (C) 2008 Wiley Periodicals, Inc. J Biomed Mater Res 87A: 780-791, 2008

Key words: BMP; drug release; calcium phosphate cement; PLGA; bone regeneration

\section{INTRODUCTION}

Calcium phosphate $(\mathrm{CaP})$ cements can be an alternative for autologous bone grafts, as these materials do not only possess favorable clinical handling properties like injectability and moldability, but also show advantageous biological characteristics like osteoconductivity and degradability. ${ }^{1-5}$ Nonetheless, these osteoconductive scaffolds alone are not sufficient for bone healing under deprived conditions, as

Correspondence to: J. A. Jansen; e-mail: j.jansen@dent. umcn.nl

(C) 2008 Wiley Periodicals, Inc. for instance in large critically sized defects. In these situations, the extra stimulatory effect of growth factor inclusion and the subsequent osteogenic capacity of $\mathrm{CaP}$ cements is an interesting approach to enhance bone regeneration.

The transforming growth factor- $\beta$ (TGF- $\beta$ ) superfamily comprises a large number of molecularly related growth and differentiation factors [TGF- $\beta$ s and bone morphogenetic proteins (BMP)], each capable of inducing bone formation..$^{6-8}$ Currently up to 20 different BMP's have been identified. ${ }^{9,10}$ These individual proteins are able to initiate the entire bone formation cascade, similar to extracts of bone matrix. ${ }^{8,11}$ Recombinant DNA biotechnology processes offer unlimited supply and control over purity and reproducibility by producing single BMP 
molecules such as BMP-2 and BMP-7..$^{8}$ The osteogenic activity of recombinant human BMP-2 (rhBMP-2) has extensively been published, and its use in humans is approved by the Food and Drug Administration Medical Device Panel. ${ }^{12}$ RhBMP-2 induces chemotaxis of stem cells and is a differentiation factor, causing mesenchymal stem cells to differentiate toward an osteoblastic lineage, providing increased numbers of osteoblasts capable of forming bone. ${ }^{8,13}$ Its effectiveness is not only dependent on this intrinsic activity, but also influenced by the method of delivery, that is, the carrier material.

Carriers for rhBMP-2 increase the retention of the protein at the treatment site for a sufficient time to allow for activity and subsequently can provide a scaffold for bone growth. ${ }^{5,14}$ Retention, that is, sustained release of rhBMP-2 is essential because BMP activity is tightly controlled and self-limiting through a combination of signal-transducing and inhibiting proteins. ${ }^{7,11}$ Consequently, bone induction is observed only locally at the site where rhBMP-2 is released and temporally when rhBMP-2 is present. This was confirmed by Woo et al., ${ }^{13}$ who showed that a sustained delivery of rhBMP-2 based on poly (DL-lactic-co-glycolic acid) (PLGA)-microspheres resulted in faster and more complete bone repair of rabbit calvarial defects as compared with immediate release of rhBMP-2. CaP cement can also act as a controlled release system by including PLGA-microparticles as delivery vehicle for rhBMP-2. ${ }^{15,16}$ The polymer will degrade over time, resulting in macropore formation and the release of rhBMP-2. The macropores will subsequently allow for cellular entry and increase the final transformation of the implant into bone.

Regarding the amount of rhBMP-2, most studies so far used relatively high nonphysiological doses, which makes treatment costly and raises the issue of safety. ${ }^{17}$ The development of better controlled release systems and the increase in knowledge of the osteoinductive effect of low doses of rhBMP-2 are therefore crucial topics for clinical application. It is assumed that the use of a carrier material for rhBMP-2, can decrease the dose of rhBMP-2 required for sufficient bone formation. ${ }^{11,18,19}$

Based on the considerations mentioned earlier, the aim of this study is to explore the bone regenerative effect of composites of CaP cement and PLGAmicroparticles with a high and a five-fold lower dose of rhBMP-2 in critically sized cranial defects in rats. In addition, the in vivo release kinetic of the two doses of rhBMP 2 were compared, using ${ }^{131} \mathrm{I}$ as a tracer. We hypothesized that a low amount of rhBMP-2 could engender sufficient bone formation in a sustained release system, resulting in closure of a critically sized defect.

\section{MATERIALS AND METHODS}

\section{Materials}

The CaP cement (Calcibon ${ }^{\circledR}$, Biomet Merck, Darmstadt, Germany) consisted of $61 \% \alpha-\mathrm{TCP}, 26 \% \mathrm{CaHPO}_{4}, \quad 10 \%$ $\mathrm{CaCO}_{3}$, and $3 \%$ precipitated $\mathrm{HA}$. The cement powder was sterilized by $\gamma$-radiation with 25 kGy (Isotron B.V., Ede, The Netherlands). The cement liquid applied was a filtersterilized $\left(0.2-\mu \mathrm{m}\right.$ filter) $1 \%$ aqueous solution of $\mathrm{Na}_{2} \mathrm{HPO}_{4}$. PLGA (Purasorb ${ }^{\circledR}$, Purac, Gorinchem, The Netherlands) with a lactic to glycolic acid ratio of 50:50 and a molecular weight of $48.0 \pm 1.6 \mathrm{~kg} / \mathrm{mol}$ was used for the microparticle preparation. RhBMP-2 was kindly provided by Wyeth Pharmaceuticals, Cambridge, MA, and radiolabeled with ${ }^{131}$ I (Amersham Buchler, Braunschweig, Germany).

\section{Radioiodination of rhBMP-2}

RhBMP-2 was labeled with ${ }^{131} \mathrm{I}$ according to the IodoGen ${ }^{\mathbb{R}}$ method as described previously. ${ }^{20}$ For each labeling procedure, $70 \mu \mathrm{g}$ of rhBMP-2 was added to $20 \mu \mathrm{L} 500 \mathrm{mM}$ phosphate buffer, $\mathrm{pH} 7.4$, and $2.25 \mathrm{mCi}{ }^{131} \mathrm{I}(200 \mathrm{mCi} / \mathrm{mL})$ in a glass vial precoated with $100 \mu \mathrm{g}$ 1,3,4,6-tetrachloro$3 \alpha, 6 \alpha$-diphenylglucouril (Pierce, Rockford, IL). The reaction mixture was incubated for $10 \mathrm{~min}$ at room temperature and then eluted on a disposable Sephadex G25M column (PD-10, Pharmacia, Uppsala, Sweden) with PBS, 0.5\% $\mathrm{BSA}$, to separate labeled rhBMP-2 from free ${ }^{131} \mathrm{I}$. The relevant fractions were pooled. The specific activity of the ${ }^{131} \mathrm{I}-$ labeled rhBMP-2 was $6.5 \mu \mathrm{Ci} / \mu \mathrm{g}$. A hot/cold ${ }^{131} \mathrm{I}-\mathrm{rhBMP}-2$ solution was prepared; 1:10 for the high dose and 1:1 for the low dose rhBMP-2 implants. The radiochemical purity of the ${ }^{131}$ I-labeled rhBMP-2 exceeded $99 \%$, and the final solution did not contain significant amounts of rhBMP-2 aggregates or degradation products. ${ }^{16,21}$

\section{Preparation of PLGA-microparticles adsorbed with rhBMP-2 or ${ }^{131}$ I-rhBMP-2}

To prepare PLGA-microparticles a double-emulsion-solvent-extraction technique ([water-in-oil]-in-water) was used. $^{15,22-25}$ This consisted of the injection of $500 \mu \mathrm{L}$ distilled water into a tube containing a solution of $1.0 \mathrm{~g}$ PLGA in $4 \mathrm{~mL}$ dichloromethane. The mixture was emulsified for $60 \mathrm{~s}$ on a vortexer. Then $6 \mathrm{~mL} 0.3 \%$ aqueous poly (vinyl alcohol) (PVA, Acros Organics, Geel, Belgium) solution was added and emulsified for another $60 \mathrm{~s}$ to produce the second emulsion. This mixture was added to $394 \mathrm{~mL}$ $0.3 \%$ PVA solution and $400 \mathrm{~mL}$ of $2 \%$ isopropylic alcohol solution and was stirred for $1 \mathrm{~h}$. The evaporation of the solvent resulted in precipitation of the dissolved polymer and subsequent microparticles were formed. The microparticles were allowed to settle for $15 \mathrm{~min}$ and the solution was decanted. Then, the microparticles were collected through centrifugation at $1500 \mathrm{rpm}$ for $5 \mathrm{~min}$, lyophilized to dryness, and stored under argon at $-20^{\circ} \mathrm{C}$ until use. The size distribution of the PLGA-microparticles was 

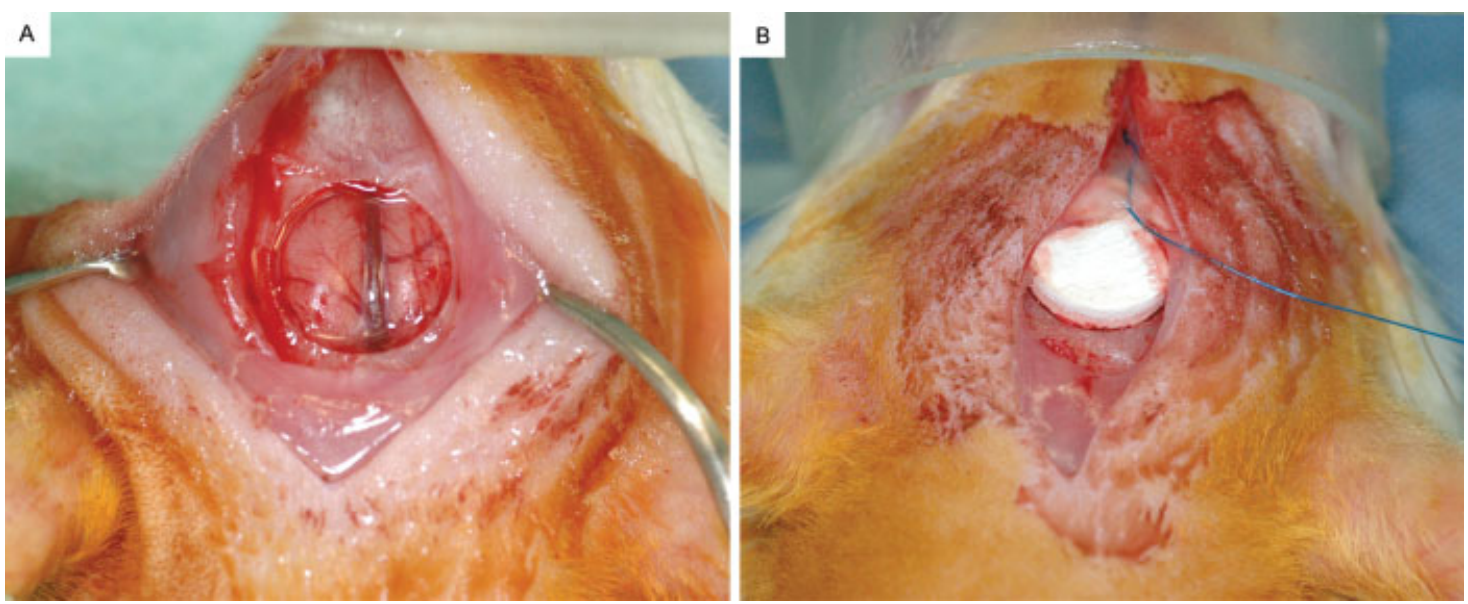

Figure 1. Cranial defect in a rat (A) with no implant and (B) with prehardened calcium phosphate cement implant. [Color figure can be viewed in the online issue, which is available at www.interscience.wiley.com.]

determined by image analysis (Leica Qwin ${ }^{\mathbb{R}}$, Leica Microsystems).

RhBMP-2 was adsorbed to the surface of the PLGAmicroparticles. Therefore $400-\mu \mathrm{L}$ rhBMP-2 solution was added to $500 \mathrm{mg}$ PLGA-microparticles, and after 30-min adsorption time the microparticles were lyophilized again. The solution for the high-dose implants $( \pm 10 \mu \mathrm{g}$ rhBMP-2 per implant) contained $275 \mu \mathrm{g}$ rhBMP-2 in PBS/BSA $[0.1 \%]$. This dose was based on previous experience with rhBMP-2 loaded PLGA/CaP cement implants in rats. ${ }^{16,21}$ For the low-dose implants $( \pm 2 \mu \mathrm{g}$ rhBMP-2 per implant) $55 \mu \mathrm{g}$ rhBMP-2 in PBS/BSA [0.1\%] was used. Accordingly, ${ }^{131}$ I-rhBMP-2 solutions were added to the PLGA-microparticles and lyophilized overnight. The adsorption efficiency of rhBMP-2 onto the microparticles was $93 \%{ }^{16}$

\section{Preparation of CaP cement implants}

CaP cement powder was mixed with blank, high $( \pm 10$ $\mu \mathrm{g}$ per disc) or low ( $\pm 2 \mu \mathrm{g}$ per disc) dose rhBMP-2, or high/low dose ${ }^{131}$ I-rhBMP-2 PLGA-microparticles (20 wt \%). The cement was created by adding to a $2-\mathrm{mL}$ syringe (BD Plastipak $^{\mathrm{TM}}$, Becton Dickinson S.A., Madrid, Spain) with closed tip, 1 wt $\% \mathrm{Na}_{2} \mathrm{HPO}_{4}$ to the PLGA/CaP powder mixture in a liquid/powder ratio of 0.35 , and shaking these components for $15 \mathrm{~s}$ using a mixing apparatus (Silamat ${ }^{\circledR}$, Vivadent, Schaan, Liechtenstein). The cement was then immediately injected into Teflon molds with standardized shape (discs $7.8 \mathrm{~mm} \times 1.8 \mathrm{~mm}$ ). After setting at room temperature overnight, the implants were removed from the molds.

\section{Surgical procedure}

Fifty-two healthy young adult male Wistar rats, weighing $256 \pm 11 \mathrm{~g}$ were included as experimental animals. National guidelines for the care and use of laboratory animals were observed. The research was reviewed and approved by the Experimental Animal Committee of the Radboud University.
Anesthesia was induced and maintained by isoflurane inhalation (Rhodia Organique Fine Limited, Avonmouth, Bristol, England). To minimize postoperative discomfort, Fentanyl ${ }^{\mathbb{R}}$ (Hameln Pharmaceuticals Gmbh, Hameln, Germany) ( $3 \mathrm{~mL} / \mathrm{kg}$ ) was administered intraperitoneally before operation and buprenorfine (Temgesic ${ }^{\mathbb{R}}$ ) (Reckitt Benckiser Health Care Limited, Schering-Plough, UK) (0.02 $\mathrm{mg} / \mathrm{kg}$ ) subcutaneously for 2 days after surgery.

After anesthesia the rats were immobilized on their abdomen, and the skull was shaved and disinfected with chloorhexidine. A longitudinal incision was made from the nasal bone to the occipital protuberance. To minimize pain, Lidocaine HCL 1\% (B. Braun, Melsungen, Germany) was dripped at the periosteum before incision and exposure of the parietal bone. A full-thickness bone defect was created in the dorsal part of the parietal cranium central to the sagittal suture with a dental drill machine (Elcomed 100, W\&H Dentalwerk Burmoos GmbH, Austria) with trephine bur (ACE dental implant system) with an outside diameter of $8.0 \mathrm{~mm}$. The bone defect was drilled under continuous saline cooling and with care for the underlying dura mater and sagittal sinus. After removing the bone segment, the defect was left empty or an implant was placed. Although CaP cement can be injected in vivo, in this study we choose for prehardened implants as the cranial defect model did not allow for injection, because of the thin bone rim and fragile underlying dura mater. The periosteal tissue was closed using nonresorbable Prolene ${ }^{\circledR}$ 5-0 (Ethicon, Somerville, New Jersey) suture material. Subsequently, the skin was closed with resorbable Vicryl ${ }^{\circledR} 4-0$ (Johnson\&Johnson, St. Stevens-Woluwe, Belgium). Implantation time was 4 or 12 weeks. The rats were divided in four groups ( $n=6$ for each implant and time period): I untreated control defects [Fig. 1(A)]; II plain CaP cement/ PLGA-microparticle implants [Fig. 1(B)]; III-IV CaP cement/PLGA-microparticle implants with respectively a high $( \pm 10 \mu \mathrm{g} /$ implant $)$ and low $( \pm 2 \mu \mathrm{g}$ /implant $)$ dose of rhBMP-2. Groups III and IV with an implantation time of 12 weeks received ${ }^{131}$ I-labeled rhBMP-2 and consisted of eight instead of six rats.

The animals were housed individually in cages. The intake of fluids and food was monitored in the first days 
postoperatively. Also, the animals were observed for signs of pain, infection, and proper activity. At the end of the implantation time the rats were sacrificed by an overdose of $\mathrm{CO}_{2}$.

\section{Scintigraphic analysis of in vivo ${ }^{131}$ I-rhBMP-2 release}

For the in vivo ${ }^{131}$ I-rhBMP-2 release study, ${ }^{16}$ rats from the 12-week implantation time group $(n=8)$ with highand low-dose ${ }^{131}$ I-rhBMP-2 implants were used. Immediately after surgery (day 0 ) and after 1, 2, 3, 4, and 5 weeks, the ${ }^{131} \mathrm{I}$ activity in the implants was determined under general isoflurane inhalation anesthesia, using a singlehead $\gamma$-camera (Orbiter, Siemens Medical Systems, Hoffman Estates, IL) equipped with a parallel-hole high-energy collimator. The analysis could not be continued after the 5 th week, because of radioactive decay. Digital images (25,000-65,000 counts) were acquired and quantitatively analyzed by measuring activity in a standardized region of interest (ROI) drawn over the implants. As a control for $100 \%$ retention, $\gamma$-emission in nonimplanted reference samples was measured along with the rat implants at each time point. The scintigraphic measurements were corrected for radioactive decay, acquisition time, and background activity. The retained activity of ${ }^{131} \mathrm{I}$-rhBMP-2 was then expressed as a percentage of the initial activity $(t=0)$. The initial dose of rhBMP-2 as present in the samples before implantation was determined by counting radioactivity with a shielded well-type $\gamma$-counter (Wizard, PharmaciaLKB, Sweden). The release of absolute amounts ( $\mu \mathrm{g})$ of rhBMP-2 out of the implanted samples was calculated from the percentage of retention.

\section{Histology and histomorphometrical analysis}

After sacrifice, implants with surrounding tissue were retrieved, fixed in phosphate-buffered formaldehyde solution $(\mathrm{pH}=7.4)$, dehydrated in increasing ethanol concentrations $(70-100 \%)$, and embedded (nondecalcified) in methylmethacrylate. Since the ${ }^{131}$ I-half-life is assumed to be 8 days, ${ }^{26}$ the implants with ${ }^{131}$ I-rhBMP-2 were also included in the histological analysis after 12 weeks of implantation. Thin sections $(10 \mu \mathrm{m})$ were prepared perpendicularly through the implants using a microtome with diamond blade (Leica Microsystems SP 1600, Nussloch, Germany). These slides were stained with methylene blue and basic fuchsin and examined with light microscopy (Leica Microsystems AG, Wetzlar, Germany).

In addition, all sections (with exception of the open defects) were quantitatively scored for the percentage of remained cement and newly formed bone using computerbased image analysis techniques (The Leica ${ }^{\mathbb{R}}$ Qwin Proimage analysis system, Wetzlar, Germany). From digitalized images (magnitude $1.6 \times$ ), the amount of remained cement and newly formed bone were determined within the ROI, that is, the area within the boundaries of the implants. The amounts were expressed as a percentage of the ROI. The measurements of three sections for each sample were averaged.

\section{Microcomputed tomography}

Before embedding in methylmethacrylate, microcomputed tomography $(\mu \mathrm{CT})$ was performed on the skulls with the empty control defects. The specimens were wrapped in Parafilm $\mathrm{M}^{\mathbb{R}}$ (Pechiney Plastic Packaging, Chicago) to prevent drying during scanning. Then all samples were scanned at an energy of $100 \mathrm{kV}$ and intensity of 98 $\mu \mathrm{A}$ with a resolution of $14.16 \mu \mathrm{m}$ using an aluminium filter (1 mm) (Skyscan-1072 X-ray microtomograph, TomoNT version 3N.5, Skyscan ${ }^{\circledR}$, Belgium). Cone-Beam reconstruction (version 2.15, Skyscan ${ }^{\mathbb{R}}$ ) was performed. All scan and reconstruction parameters applied were identical for all specimens. Three-dimensional images were created using 3D-creator (version 2.2h, Skyscan ${ }^{\mathbb{R}}$ ) and evaluated for the percentage of defect closure. The data were then further analyzed by CT Analyzer (version 1.4, Skyscan ${ }^{\mathbb{R}}$ ). The volume of interest (VOI) was specified as a cylindrical area covering the created defect with a diameter of $8.02 \mathrm{~mm}$ and depth of $1.2 \mathrm{~mm}$. In this area, bone volume $\left(\mathrm{mm}^{3}\right)$ was determined and expressed as a percentage of the VOI.

\section{Statistical Analysis}

Statistical analysis was performed with GraphPad Instat version 3.05 (GraphPad Software, San Diego, CA), using an unpaired $t$-test with Welch correction. Differences were considered significant at $p$-values less than 0.05. Pharmacokinetic analysis of rhBMP-2 retention was done by nonlinear regression analysis using GraphPad ${ }^{\circledR}$ Prism 4.03 software (GraphPad Software, San Diego, CA). Data were fitted according to a one-phase exponential decay model.

\section{RESULTS}

\section{Characterization of PLGA-microparticles}

Figure 2 shows the size distribution of the PLGAmicroparticles prepared by a double-emulsionsolvent-extraction technique. The average size of the microparticles was $33 \pm 28 \mu \mathrm{m}$, as determined with image analysis. Scanning electron micrographs (SEM; Microscopic Imaging Center of the Nijmegen

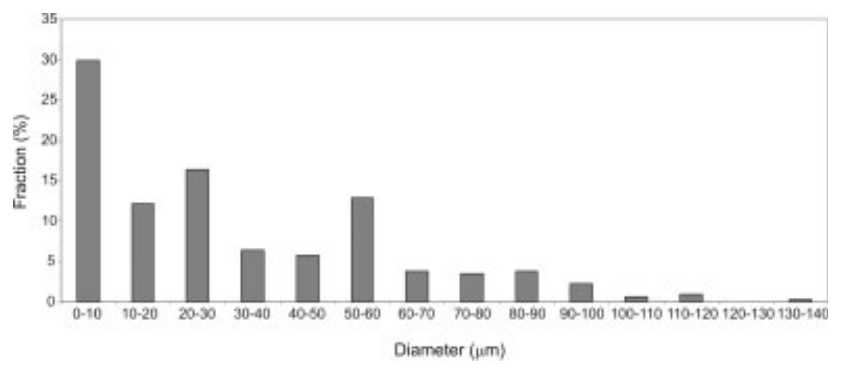

Figure 2. Size distribution of the PLGA-microparticles prepared by a double-emulsion-solvent-extraction technique (group size $=312$ ). 

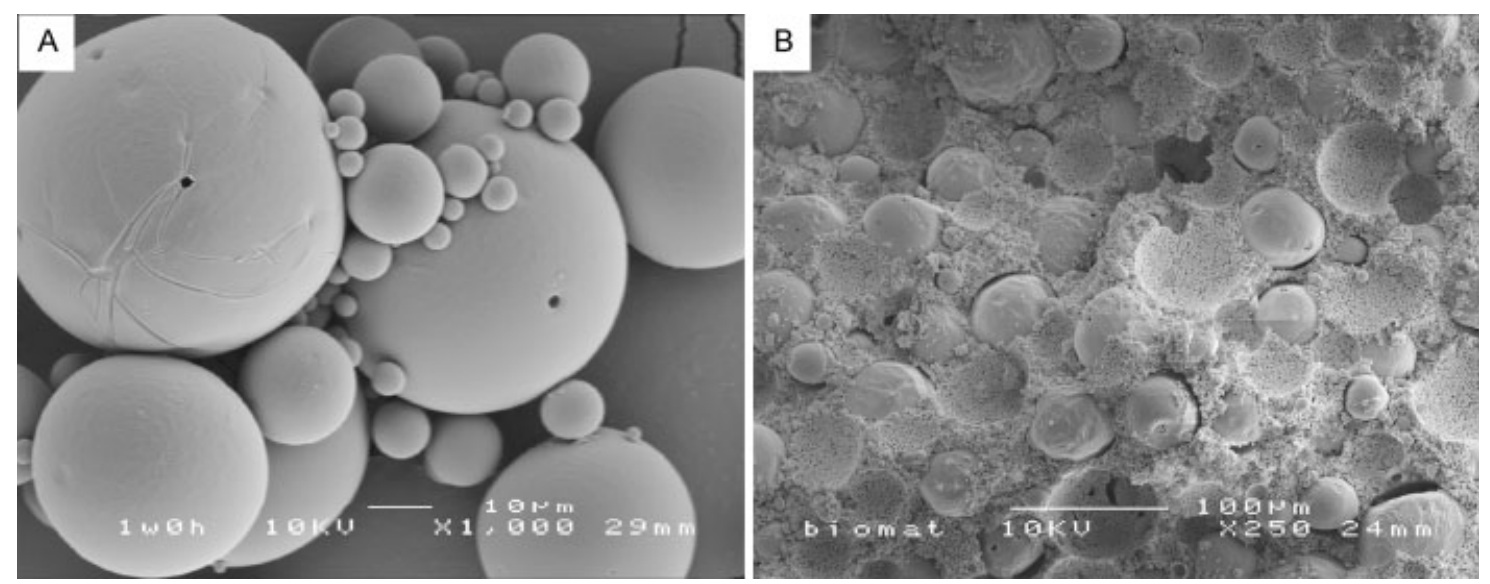

Figure 3. Scanning electron micrograph of (A) PLGA-microparticles alone and (B) incorporated in CaP cement.

Center for Molecular Life Sciences, The Netherlands) revealed a smooth spherical surface of the microparticles, occasionally with pores [Fig. 3(A)]. The microparticles were well incorporated and homogeneously divided into the cement [Fig. 3(B)].

\section{Longitudinal in vivo ${ }^{131}$ I-rhBMP-2 release}

Longitudinal release kinetic over 5 weeks of implantation was assessed by scintigraphic imaging of the radiolabeled implants in vivo. All animals, except one, underwent the scintigraphic procedure well. One rat (high dose ${ }^{131}$ I-rhBMP-2 implant) died after 4 weeks of scintigraphic measurements, most likely because of an anesthetic-induced respiratory distress. Consequently, the high-dose group consisted of seven instead of eight rats from 5 weeks onward.

The results of the ${ }^{131}$ I-rhBMP-2 retention of the high- and low-dose implants are depicted in Figure 4(A). Both doses showed a sustained release profile, with a considerable retention (about $70 \%$ ) of rhBMP2 after 5 weeks of implantation. The percentage of retention in the low-dose implants was significantly lower $(p<0.01)$ as compared with the high-dose implants in the first 4 weeks of implantation. The data of the low-dose implants could be described best according to a one-phase exponential release model, with a half-life of $0.6 \pm 0.1$ weeks and plateau value of $67.9 \% \pm 1.4 \%$. The high-dose implants, on the other hand, displayed an almost linear release of rhBMP-2 within the first 5 weeks of implantation, which is suggestive for a process approaching zeroorder kinetics. Nevertheless, we fitted the one-phase exponential model also to the high-dose implant data, but the calculated half-life of $7.4 \pm 8.4$ weeks was necessarily less accurate and a reliable estimate for the plateau value could not be made.

The initial dose of rhBMP-2 as present in the samples before implantation was determined by count- ing the activity in the implants in a shielded welltype $\gamma$-counter. The high-dose implants contained a initial dose of $8.2 \pm 1.8 \mu \mathrm{g}$ rhBMP-2, the low-dose implants $1.7 \pm 0.4 \mu \mathrm{g}$. Figure $4(\mathrm{~B})$ reveals the absolute cumulative release of rhBMP-2 in the first 5 weeks of implantation. The lines through the data

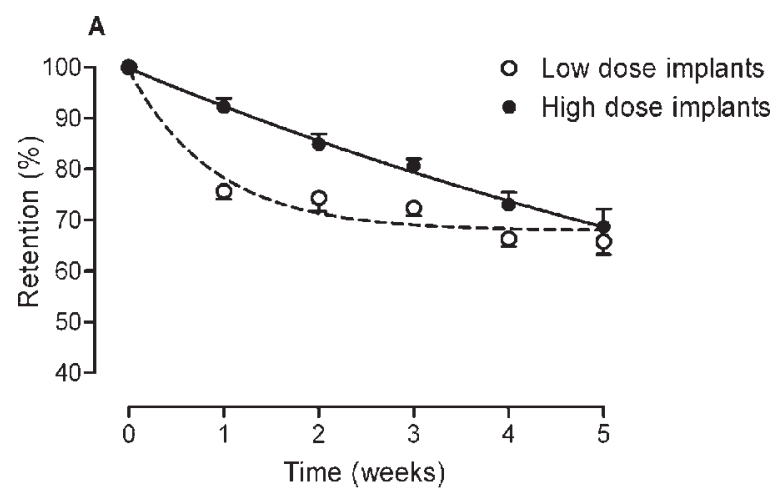

B

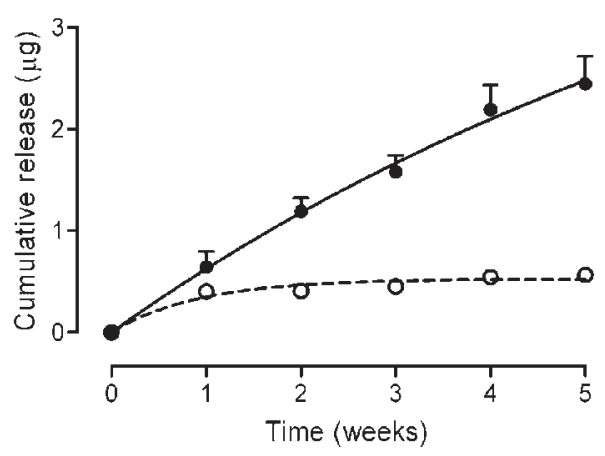

Figure 4. Longitudinal scintigraphic assessment of a high and low dose of rhBMP-2 (A) retained in the implants (\%) and $(\mathrm{B})$ the absolute cumulative release of rhBMP-2 $(\mu \mathrm{g})$ out of $\mathrm{CaP}$ cement implants. Mean and standard error of the mean are presented for eight samples. The percentage of retention in the low dose implants was significantly lower ( $p<0.01$, unpaired $t$-test with Welch correction) as compared with the high dose implants in the first 4 weeks of implantation. 


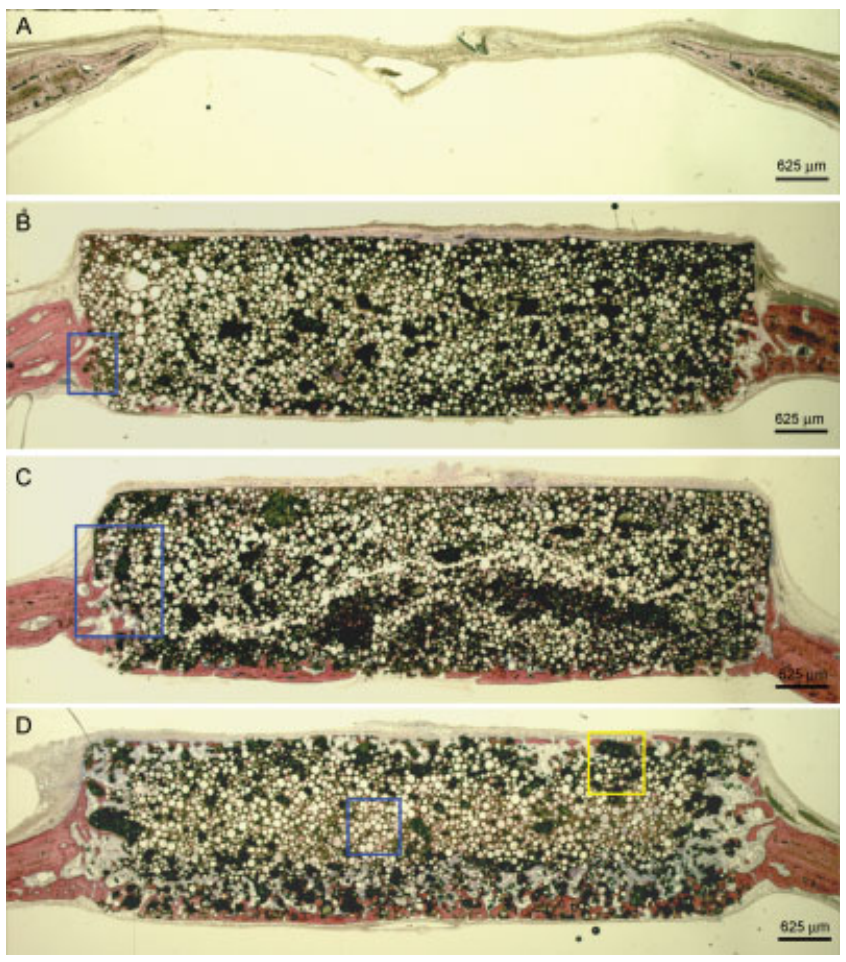

Figure 5. Representative histological sections of cranial defects after 4 weeks of implantation with (A) no implant, (B) plain-CaP cement implant, (C) low-dose rhBMP-2 CaP cement implant, and (D) high-dose rhBMP-2 CaP cement implant. Methylene blue and basic fuchsine staining. Magnifications of the boxes are depicted in Figure 6. [Color figure can be viewed in the online issue, which is available at www.interscience.wiley.com.]

points were again calculated according to a onephase exponential release model. The high-dose group showed a higher release of rhBMP-2 (2.4 \pm $0.8 \mu \mathrm{g})$ after 5 weeks as compared with the low-dose implants $(0.6 \pm 0.2 \mu \mathrm{g})$.

As a control for $100 \%$ retention, nonimplanted reference samples were scanned simultaneously with the rats. These eight reference samples showed an average retention of $94 \pm 2 \%$ over the 5 -week period. This decrease in retention indicates that the measured release values are somewhat overestimated.

\section{Descriptive light microscopy}

A uniform tissue reaction was seen between the specimens of the same group and implantation time.

Implantation time 4 weeks

The skulls that had received no implant showed minimal bone formation at the defect borders and fibrous tissue crossing the defect area [Fig. 5(A)].
In the skulls that received an implant [Fig. 5(BD)], the integrity of the implants was maintained for all formulations. Minimal edge degradation was seen in the plain- and low-dose implants, while in the high-dose rhBMP-2 group, the implant perimeter appeared to be more rounded. The PLGA pores located at the external surface of the implants were filled with bone, fibrous tissue, or bone marrow-like tissue [Fig. 6(B)]. However, in the center of the implant remnants of PLGA were still visible in the pores, characterized by stained material without cells [Fig. 6(C)]. In general, a mild inflammatory reaction was seen as macrophages and foreign body giant cells were only occasionally present.

Bone ingrowth was found at the edges and dura side of the implants of all formulations, with a direct contact of bone to cement surface [Fig. 6(A)]. Bone formation was higher in implants with rhBMP-2 as compared with plain implants. Moreover, in the rhBMP-2 loaded implants and mainly in the highdose group, new bone was also seen at the periosteum side [Fig. 6(D)]. In the loaded groups, bone remodeling was higher as compared with the plain implants. In these groups, osteoclast-like cells were observed degrading the implant material, while directly opposite new bone was deposed. Bone bridging of the defect, as characterized by a continuous thin bone layer at the dura side of the skull, was found in none of the plain implants, three out of six low dose and four out of six high rhBMP-2 dose implants.

Implantation time 12 weeks

After 12 weeks of implantation, the skulls with empty control defects only showed bone formation at the defect borders [Fig. 7(A)], confirming their critical size.

The groups that received plain-and low-dose implants [Fig. 7(B,C)] showed some degradation with rounded implant edges. The high-dose implants [Fig. 7(D)], on the other hand, were clearly degraded superficially. In the periphery, ingrowth of tissue had proceeded into the PLGA pores of the implants, while in the center no cells were visible [Fig. 8(A)].

Bone formation was increased for all formulations as compared with the 4-week sections, with the largest amount of bone in the high-loaded implants. Mature bone with bone marrow-like tissue was deposed directly to the cement surface [Fig. 8(B)]. Bridging at the dura side was observed in two plain, three low dose, and four high dose implants out of totally six implants per group. In addition, the highdose rhBMP-2 implants also showed new bone on the periosteum side [Fig. $8(\mathrm{C})$ ]. 

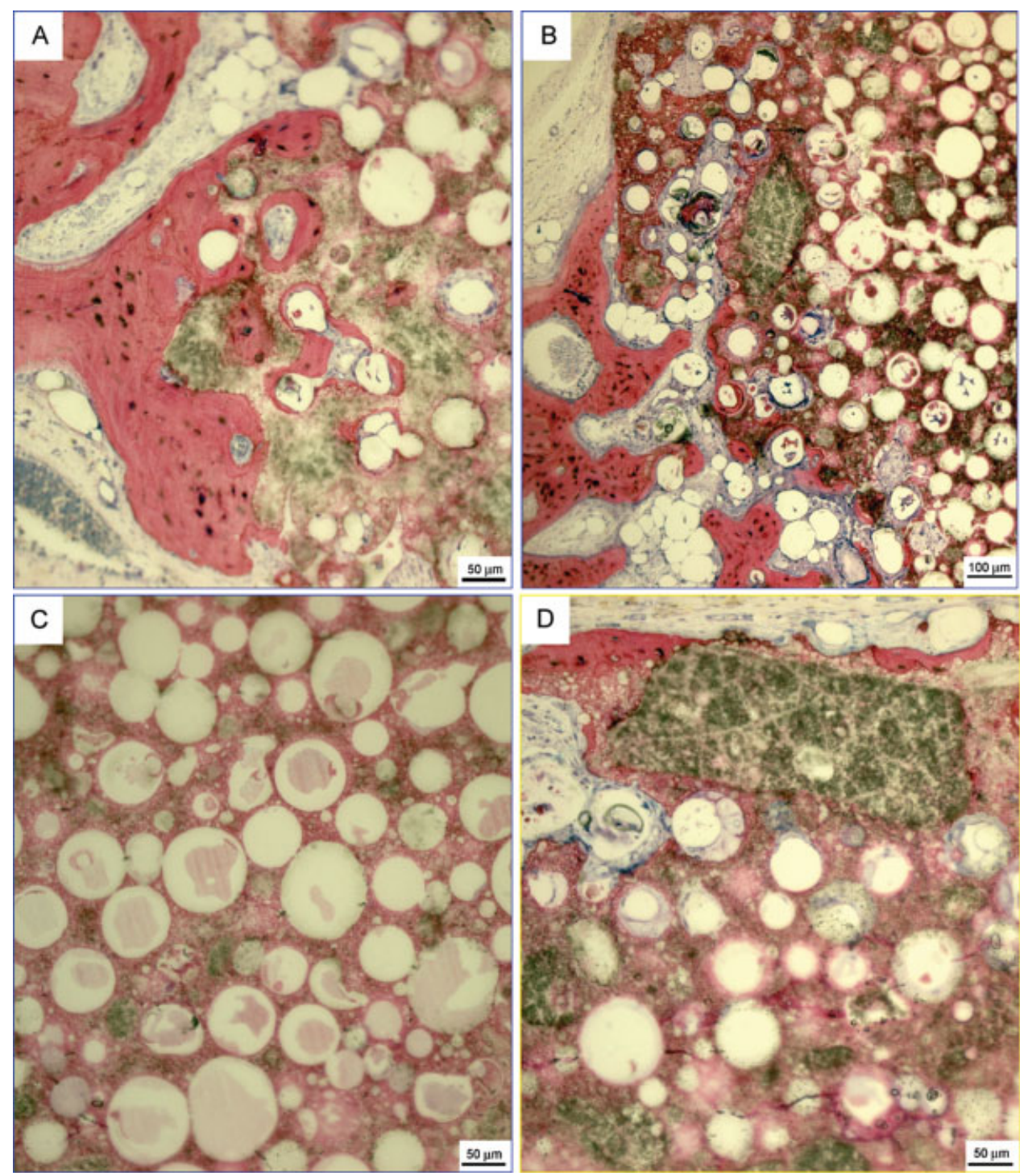

Figure 6. Magnifications of the boxes depicted in (A) Figure 5(B), showing direct bone-cement contact; (B) Figure 5(C), at the edge of the implant; (C, D) Figure 5(D), of respectively the center and periosteum side of the implant. Methylene blue and basic fuchsine staining. [Color figure can be viewed in the online issue, which is available at www.interscience.wiley. com.]

Histomorphometry

The results of the quantitative measurements of remained $\mathrm{CaP}$ cement and the amount of bone formed into the implants are depicted in Tables I and II, respectively.

After 4 weeks of implantation no significant differences in implant degradation were found between the groups. After 12 weeks, all type of implants showed degradation when compared with the 4week implantation time, though this was only significant $(p=0.04)$ for the high-dose group, with $49 \%$ $\pm 2 \%$ of implant remained in the ROI.

Bone formation was significantly $(p<0.01)$ higher in the implants with rhBMP-2 (low and high dose) as compared with the plain implants after 4 weeks im- plantation. No difference in percentage of bone was seen between the two doses at this time point. Bone formation increased significantly in the plain- and high-rhBMP-2 implants after 4 weeks $(p<0.01)$. After 12 weeks, bone formation was significantly $(p=0.01)$ higher in the high-dose group when compared with the plain implants. There was a tendency of more bone formation in the high dose as compared with the low-dose implants (95\% confidence interval -0.35 to 8.98; $p=0.06$, unpaired $t$-test with Welch correction).

\section{MicroCT}

MicroCT scanning was performed on the skulls that received no implant to check whether the cre- 

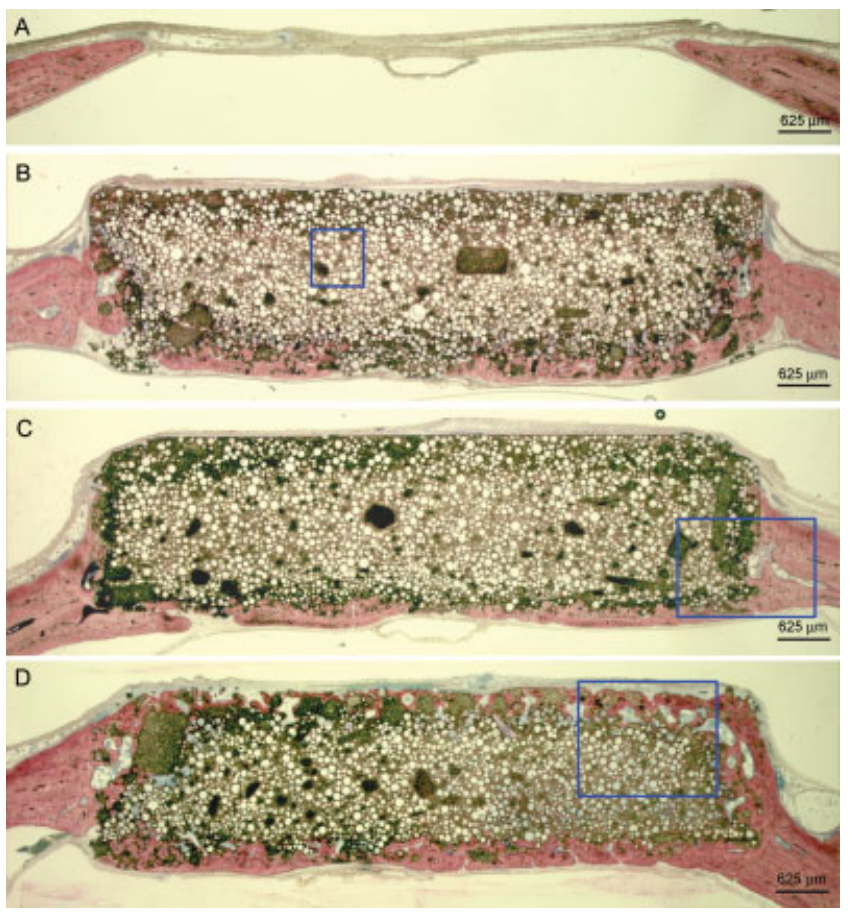

Figure 7. Representative histological sections of cranial defects after 12 weeks of implantation with (A) no implant, (B) plain-CaP cement implant, (C) low-dose rhBMP-2 CaP cement implant, and (D) high-dose rhBMP-2 CaP cement implant. Methylene blue and basic fuchsine staining. Magnifications of the boxes are depicted in Figure 8.

ated defect was of critical-size after 4 and 12 weeks implantation. The results are shown in Figure 9. Four and 12 weeks after implantation no defect bridging was noticed in all specimens, and less than $10 \%$ bone had formed indicating that the defects were indeed of critical size.

\section{DISCUSSION}

A reduction in the amount of rhBMP-2 required for sufficient bone regeneration is beneficial for bone graft substitutes from a socioeconomical and a safety point of view. In this study, implants consisting of composites of $\mathrm{CaP}$ cement and PLGA-microparticles and a high and five-fold lower dose of rhBMP-2 were compared for their kinetics and bone regenerative behavior in critically sized cranial defects in rats. ${ }^{131}$ I-labeled rhBMP-2 showed for both doses a sustained release pattern within the first 5 weeks, with different release profiles between the two loadings. About $70 \%$ of rhBMP-2 remained in both implant formulations. Despite the low amount of released rhBMP-2, bridging of critically sized defects was seen after 12 weeks of implantation in the highloaded implants.

RhBMP-2 has strong osteoinductive potential but its clinical use is restrained by the lack of a suitable delivery system, that maintains rhBMP-2 at the defect site for sufficient time and with appropriate dose to allow for chemotaxis of preosteoblastic cells and their differentiation into bone forming cells. ${ }^{13,27}$ A sustained release system complying to these requirements, might be obtained using composites of
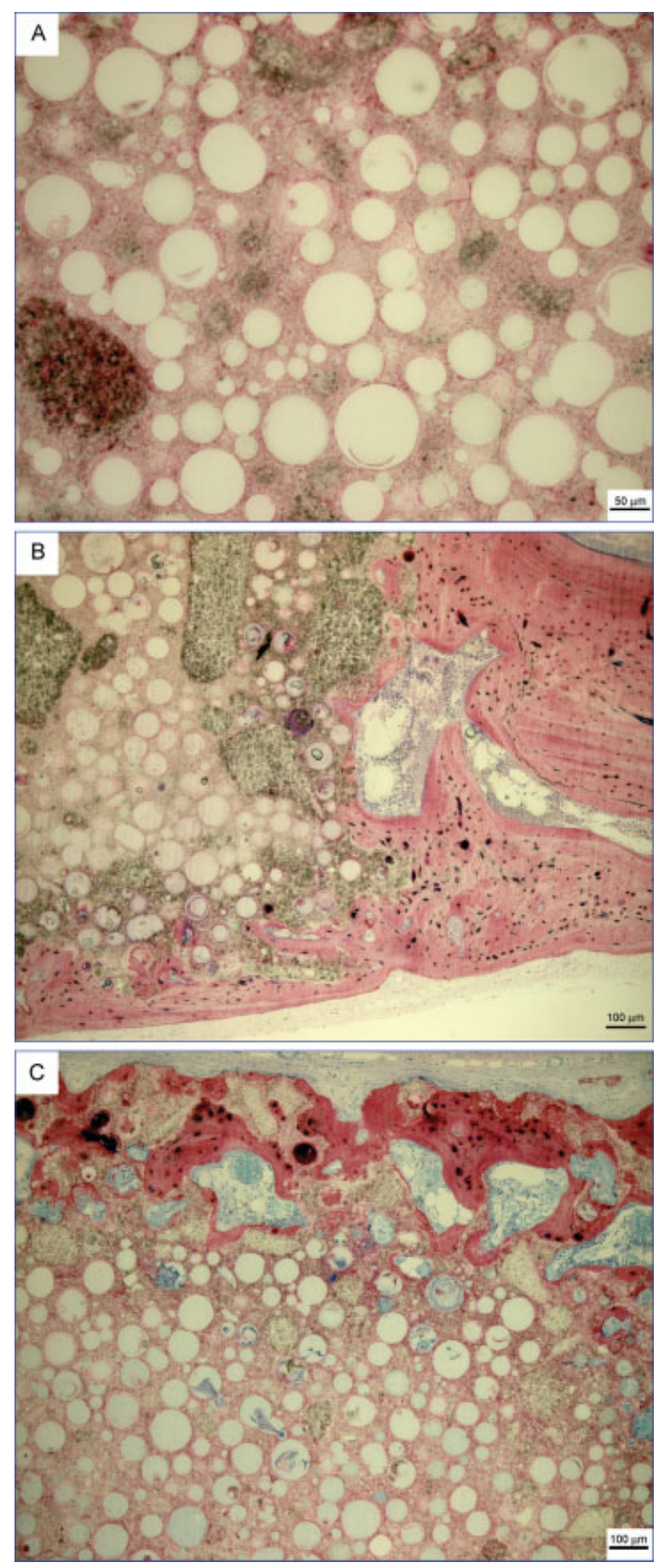

Figure 8. Magnifications of the boxes depicted in (A) Figure 7(B), showing the center of the implant; (B) Figure 7(C), at the edge of the implant; (C) Figure 7(D), of the periosteal side of the implant. Methylene blue and basic fuchsine staining. 
TABLE I

The Percentage of Remained Implant in the ROI as Determined with Histomorphometry

\begin{tabular}{lccc}
\hline & & \multicolumn{2}{c}{ RhBMP-2 Implant } \\
\cline { 3 - 4 } \% Implant & Plain Implant & Low Dose & High Dose \\
\hline 4 weeks & $58 \pm 4$ & $59 \pm 3$ & $56 \pm 6$ \\
12 weeks & $55 \pm 3$ & $55 \pm 5$ & $49 \pm 2^{*}$ \\
\hline
\end{tabular}

Data represent the mean and standard deviations. Highdose rhBMP-2 implants significantly $\left({ }^{*}\right)$ degraded after 12 weeks of implantation (unpaired t-test with Welch correction).

$\mathrm{CaP}$ cement and rhBMP-2 loaded polymeric microparticles. The injectable and moldable $\mathrm{CaP}$ cement acts as a scaffold for bone growth and because of polymer degradation osteoinductive rhBMP-2 is gradually released. Subsequent to microparticle degradation, macropores are formed which allow for cellular entry, followed by transformation of the implant into bone tissue. This study, indeed revealed a sustained release of rhBMP-2 in the high- and low-dose CaP cement/PLGA-microparticle implants. This finding is in line with the study of Ruhe et al., ${ }^{16}$ who also showed in vivo sustained release of rhBMP-2 from subcutaneous CaP cement composites when entrapped or adsorbed to PLGA-microparticles, in rats.

Remarkably, the amount of rhBMP-2 that was retained in the implants, expressed as a percentage of the initial dose, was significantly lower in the low dose than in the five-fold higher dose group in the first 4 weeks and especially in the first week of implantation. RhBMP-2 release in the first days of implantation is thought to derive from superficially located PLGA-microparticles and to a lesser extent

TABLE II

The Percentage of New Bone in the ROI as Determined with Histomorphometry

\begin{tabular}{lccc}
\hline \multirow{2}{*}{$\%$ Bone } & Plain Implant & Low Dose & High Dose \\
\cline { 3 - 4 } & $5.5 \pm 0.4$ & $8.2 \pm 1.3^{*}$ & $8.1 \pm 1.3^{*}$ \\
\hline w weeks & $10 \pm 3^{*}$ & $11 \pm 4$ & $16 \pm 2^{* *}$ \\
\hline
\end{tabular}

Data represent the mean and standard deviations. After 4 weeks, the percentage of bone in the low- and high-dose rhBMP-2 implants was significantly $\left(^{*}\right)$ higher as compared with plain implants. After 12 weeks, the difference between plain- and high-dose rhBMP-2 implants was significant $\left({ }^{* *}\right)$, and there was a tendency $(p=0.06)$ of more bone formation in the high as compared with the low-dose rhBMP-2 implants. Comparing the 4- and 12-week implantation time, plain- and high-dose implants showed a significant $\left(^{*}\right)$ increase in percentage of bone (unpaired $t$-test with Welch correction).

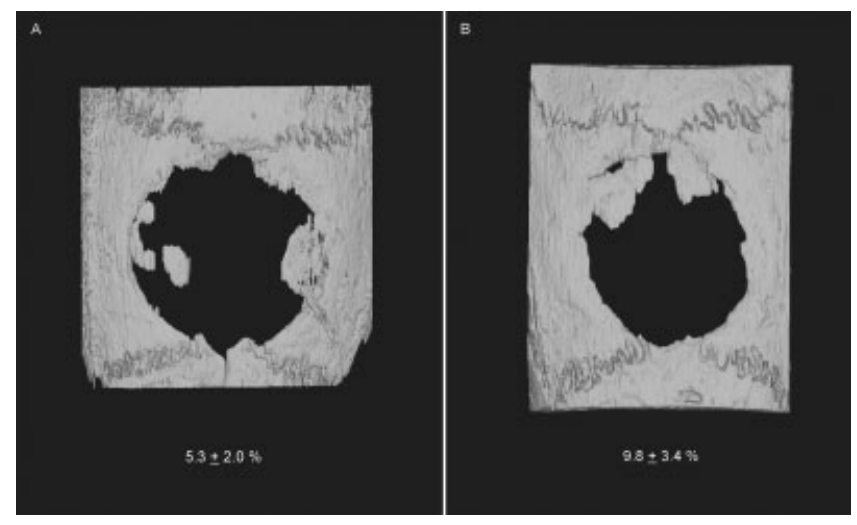

Figure 9. Micro-CT images of a cranial defect $(8 \mathrm{~mm}$ after (A) 4 weeks and (B) 12 weeks of implantation, with the percentage of bone formed in the defect depicted below.

from CaP cement located at the implant surface, as in vitro studies showed that release from PLGAmicroparticles is significantly higher than from $\mathrm{CaP}$ cement composites. ${ }^{15}$ The difference in percentage of retention between the two groups in this study might be explained by the PLGA-microparticle adsorption efficiency of low and high doses of rhBMP-2. It can be speculated that low doses of rhBMP-2 bind stronger to microparticles than high doses, as a high dose leads to saturation of binding to the microparticles and subsequent translocation of rhBMP-2 to CaP cement. Also electrostatic interactions might explain the difference in retention profiles between high and low amounts of rhBMP-2. ${ }^{28}$ RhBMP-2 was diluted in a solution containing BSA to which it can bind, and the resulting complex is negatively charged. Low doses of rhBMP-2 are surrounded by higher amounts of BSA than high doses, and will thereby be easily released from negatively charged $\mathrm{CaP}$ cement implants. More detailed studies are needed to determine the mechanism of this complex phenomenon.

After 1 week, retention in the low-loaded implants was more or less stabilized, while the high-dose implants showed an almost linear release, approaching the characteristics of zero-order kinetics. The relatively short half-life of ${ }^{131} \mathrm{I}(8.02 \text { days })^{26}$ allowed histological analysis of the implants in the same rats that received radiolabeled rhBMP-2. The histological analyses at 4 weeks implantation indicated that $\mathrm{CaP}$ cement degradation was higher in the high-loaded group, supporting the suggestion that rhBMP-2 release from 1 week on was degradation dependent. So far we do not know any previous studies that have dealt with the kinetics of differently loaded $\mathrm{CaP}$ cement implants, and that were able to combine release and histological data. The influence of rhBMP-2 to implant degradation, which was mainly seen in the high-dose group, is consistent with previous investigations and might be explained by the 
regulation of osteoclastic precursors by the biological action of rhBMP-2 $2^{29,30}$ or by new bone marrow formation where osteoclastic precursors reside. ${ }^{17}$

The implants were placed in $8-\mathrm{mm}$ cranial defects in rats. Untreated defects clearly showed to be critically sized, which corroborates with previous studies. ${ }^{31,32}$ Plain composites of CaP cement/PLGAmicroparticles improved bone growth by providing an osteoconductive scaffold, but the combination of osteoconduction and osteoinductive rhBMP-2 turned out to be capable to bridge the critical defect. Bone formation in all implant formulations was not only located at the defect edges, but also at the dura side. This is consistent with preceding investigations, ${ }^{31,32}$ demonstrating that the dura mater delivers precursor cells involved in rhBMP-2-induced bone formation. In addition, Wang and Glimcher ${ }^{32}$ performed ${ }^{3} \mathrm{H}$-thymidine labeling studies, showing that subsequent to the dura mater, preosteoblasts were derived from the connective tissue covering the defects. In this study, such periosteal bone formation was only seen in the high-loaded implants.

The main aim of this study was to examine whether a low dose of rhBMP-2 could be as effective in bone regeneration as a generally used higher dose, ${ }^{6,27,33-37}$ when released in a sustained manner. As mentioned earlier, this study allowed for the combination of release and histological data. Histology showed significantly more bone in the implants loaded with a high than with a low dose of rhBMP-2 after 12 weeks. Nevertheless, only $2.4 \pm 0.8 \mu \mathrm{g}$ rhBMP-2 was released in 5 weeks of implantation. The release profile thereafter could not be measured, but is supposed to follow implant degradation. At 12 weeks of implantation, degradation of the highloaded implants was limited to about $50 \%$, which confirms that the total released dose did not reach 5 $\mu \mathrm{g}$, and that high doses of rhBMP-2 are not a prerequisite to obtain enhanced bone regeneration. The dose required for improved bone regeneration should overcome the tight regulation of osteogenic and inhibiting factors 7,11 and should trigger sufficient amounts of BMP responsive cells, that is the critical cell density, that can generate the desired bone formation. ${ }^{14}$ Apparently, our high-dose implants complied with these necessities. The lowdose implants, on the other hand, showed significantly more bone formation after 4 weeks implantation as compared with the plain implants. However, this difference disappeared after 12 weeks, suggesting that the critical cell density was not reached and that the required dose for enhanced bone formation is bound to a threshold. Although this study shows that low amounts of rhBMP-2 are sufficient to increase bone regeneration, no clear extrapolation of absolute values as required in larger animal models and humans can be made, because higher animal species need larger doses of rhBMP-2 to obtain equal effects as in rodents. ${ }^{5,6,11,14}$ Nonetheless, this study provides a proof of principle that the required relative amounts of rhBMP-2 can be reduced by using a sustained release system, as we were able to identify the amounts of rhBMP-2 that were actually released and to combine these data with histology.

It has to be noted that the rhBMP-2 release as assessed by scintigraphic imaging is somewhat overestimated, as the nonimplanted reference samples showed a decrease in retention. However, longitudinal scintigraphy was found to be reliable in previous studies, ${ }^{16,21}$ and the overestimation of rhBMP-2 release does not conflict with the conclusion that low doses of rhBMP-2 are capable of inducing enhanced bone formation.

Both implant formulations and the low-dose group in particular, showed a considerable retention of rhBMP-2. This might be explained by the high binding affinity of rhBMP-2 for CaP cement, ${ }^{5,33}$ but also by the limited PLGA-microparticle degradation. In this study, no cells were found in the center of the implants after 4 and 12 weeks of implantation. This is in contrast with the study of Ruhé et al., ${ }^{38}$ who used smaller implants of the same material, placed subcutaneously, and in noncritically sized cranial defects in rats. Throughout these implants tissue ingrowth was seen, confirming degradation of the PLGA. Evidently, in our critically sized samples PLGA was entrapped in the center of the implant and thereby withdrawn from necessary fluids for degradation, which may explain the limited cellular entry. Besides larger implant size, in this study the dimension of the microparticles was also smaller as compared with the samples in the study of Ruhé et al. (33 $\pm 28 \mu \mathrm{m}$ and $66 \pm 25 \mu \mathrm{m}$, respectively). This narrow pore diameter may have hindered new tissue infiltration. In conclusion, although this study revealed that low amounts of rhBMP-2 are sufficient to enhance bone regeneration, high amounts of rhBMP-2 were retained in the CaP cement implants. Complete release of rhBMP-2 and improved implant degradation has to be the goal of future studies, which perhaps can be obtained by using more rapidly degrading low-molecular weight PLGA-microparticles or particles made from other polymers.

A complex factor in rhBMP-2 bioactivity concerns the way the protein becomes activated. It has been assumed that rhBMP-2 not only acts after being released, but also when it is presented to the target cells in a "bound" form. ${ }^{39-41}$ According to this theory, retained surface bound rhBMP-2 could have influenced the amount of bone formation in this study. The extent of this contribution is difficult to determine, as no techniques are available to differentiate between bound and released forms of rhBMP-2 in vivo. 


\section{CONCLUSIONS}

PLGA/CaP cement composites are sustained release carriers for rhBMP-2, in which the loading of rhBMP-2 influences the release profile and the release is degradation dependent. In this sustained release system, the controlled release of a low dose $(2.4 \pm 0.8 \mu \mathrm{g}$ in 5 weeks) of rhBMP-2 was found to be sufficient to induce bone bridging of critically sized skull defects. Relatively high amounts of rhBMP-2 retained in the composites, because of the slow release rate and limited PLGA-microparticle degradation.

The authors thank Cathelijne Frielink and Huub Rennen for their help with the ${ }^{131}$ I-rhBMP-2 release study. RhBMP2 was kindly provided by Wyeth Pharmaceuticals (Cambridge, MA).

\section{References}

1. del Real RP, Ooms E, Wolke JG, Vallet-Regi M, Jansen JA. In vivo bone response to porous calcium phosphate cement. J Biomed Mater Res A 2003;65:30-36.

2. Ooms EM, Wolke JG, van der Waerden JP, Jansen JA. Trabecular bone response to injectable calcium phosphate (Ca-P) cement. J Biomed Mater Res 2002;61:9-18.

3. Yuan H, Li Y, de Bruijn JD, de Groot K, Zhang X. Tissue responses of calcium phosphate cement: A study in dogs. Biomaterials 2000;21:1283-1290.

4. Jansen J, Ooms E, Verdonschot N, Wolke J. Injectable calcium phosphate cement for bone repair and implant fixation. Orthop Clin North Am 2005;36:89-95.

5. Ginebra MP, Traykova T, Planell JA. Calcium phosphate cements as bone drug delivery systems: A review. J Control Release 2006;113:102-110.

6. Groeneveld EH, Burger EH. Bone morphogenetic proteins in human bone regeneration. Eur J Endocrinol 2000;142:9-21.

7. Ebara S, Nakayama K. Mechanism for the action of bone morphogenetic proteins and regulation of their activity. Spine 2002;27 (Suppl):s10-s15.

8. Wozney JM. Overview of bone morphogenetic proteins. Spine 2002;27 (Suppl):s2-s8.

9. Chen D, Zhao M, Mundy GR. Bone morphogenetic proteins. Growth Factors 2004;22:233-241.

10. Cao $\mathrm{X}$, Chen D. The BMP signaling and in vivo bone formation. Gene 2005;357:1-8.

11. Seeherman H, Wozney J, Li R. Bone morphogenetic protein delivery systems. Spine 2002;27 (Suppl):s16-s23.

12. Rodeo SA. What's new in orthopaedic research. J Bone Joint Surg Am A 2003;85:2054-2062.

13. Woo BH, Fink BF, Page R, Schrier JA, Jo YW, Jiang G, DeLuca M, Vasconez HC, DeLuca PP. Enhancement of bone growth by sustained delivery of recombinant human bone morphogenetic protein-2 in a polymeric matrix. Pharm Res 2001;18:1747-1753.

14. Seeherman H, Wozney JM. Delivery of bone morphogenetic proteins for orthopedic tissue regeneration. Cytokine Growth Factor Rev 2005;16:329-345.

15. Ruhe PQ, Hedberg EL, Padron NT, Spauwen PH, Jansen JA, Mikos AG. rhBMP-2 release from injectable poly(DL-lactic-coglycolic acid)/calcium-phosphate cement composites. J Bone Joint Surg Am A 2003;85 (Suppl):s75-s81.
16. Ruhe PQ, Boerman OC, Russel FG, Spauwen PH, Mikos AG, Jansen JA. Controlled release of rhBMP-2 loaded poly(DLlactic-co-glycolic acid)/calcium phosphate cement composites in vivo. J Control Release 2005;106:162-171.

17. Hoshino M, Egi T, Terai H, Namikawa T, Takaoka K. Repair of long intercalated rib defects using porous $\beta$-tricalcium phosphate cylinders containing recombinant human bone morphogenetic protein-2 in dogs. Biomaterials 2006;27:4934-4940.

18. Kamakura S, Nakajo S, Suzuki O, Sasano Y. New scaffold for recombinant human bone morphogenetic protein-2. J Biomed Mater Res A 2004;71:299-307.

19. Barnes B, Boden SD, Louis-Ugbo J, Louis-Ugbo J, Tomak PR, Park JS, Park MS, Minamide A. Lower dose of rhBMP-2 achieves spine fusion when combined with an osteoconductive bulking agent in non-human primates. Spine 2005;30: 1127-1133.

20. Fraker PJ, Speck JC Jr. Protein and cell membrane iodinations with a sparingly soluble chloroamide, 1,3,4,6-tetrachloro-3a,6adiphrenylglycoluril. Biochem Biophys Res Commun 1978;80: 849-857.

21. Ruhe PQ, Boerman OC, Russel FG, Mikos AG, Spauwen PH, Jansen JA. In vivo release of rhBMP-2 loaded porous calcium phosphate cement pretreated with albumin. J Mater Sci: Mater Med 2006;17:919-927.

22. Habraken WJEM, Wolke JGC, Mikos AG, Jansen JA. Injectable PLGA microsphere/calcium phophate cements: Physical properties and degradation characteristics. J Biomater Sci Polym Ed 2006;17:1057-1074.

23. Lu L, Stamatas GN, Mikos AG. Controlled release of transforming growth factor $\beta 1$ from biodegradable polymer microparticles. J Biomed Mater Res 2000;50:440-451.

24. Peter SJ, Lu L, Kim DJ, Stamatas GN, Miller MJ, Yaszemski MJ, Mikos AG. Effects of transforming growth factor $\beta 1$ released from biodegradable polymer microparticles on marrow stromal osteoblasts cultured on poly(propylene fumarate) substrates. J Biomed Mater Res 2000;50:452-462.

25. Cleek RL, Rege AA, Denner LA, Eskin SG, Mikos AG. Inhibition of smooth muscle cell growth in vitro by an antisense oligodeoxynucleotide released from poly(DL-lactic-co-glycolic acid) microparticles. J Biomed Mater Res 1997;35:525-530.

26. Burkinshaw L. The half-life of iodine 131. Phys Med Biol 1958;2:255-257.

27. Arnaud E, De Pollak C, Meunier A, Sedel L, Damien C, Petite $\mathrm{H}$. Osteogenesis with coral is increased by BMP and BMC in a rat cranioplasty. Biomaterials 1999;20:1909-1918.

28. Andrade JD, Hlady V, Feng L, Tingey K. Interfacial Phenomena and Bioproducts. New York: Marcel Dekker; 1996. pp 19-55.

29. Seeherman HJ, Azari K, Bidic S, Rogers L, Li XJ, Hollinger JO, Wozney JM. rhBMP-2 delivered in a calcium phosphate cement accelerates bridging of critical-sized defects in rabbit radii. J Bone Joint Surg Am 2006;88:1553-1565.

30. Itoh K, Udagawa N, Katagiri T, Iemura S, Ueno N, Yasuda H, Higashio K, Quinn JM, Gillespie MT. Bone morphogenetic protein 2 stimulates osteoclast differentiation and survival supported by receptor activator of nuclear factor- $\kappa \mathrm{B}$ ligand. Endocrinology 2001;142:3656-3662.

31. Takagi K, Urist MR. The reaction of the dura to bone morphogenetic protein (bmp) in repair of skull defects. Ann Surg 1982;196:100-109.

32. Wang J, Glimcher MJ. Characterization of matrix-induced osteogenesis in rat calvarial bone defects. II. Origins of boneforming cells. Calcif Tissue Int 1999;65:486-493.

33. Jansen JA, Vehof JW, Ruhe PQ, Kroeze-Deutman H, Kuboki Y, Takita H, Hedberg EL, Mikos AG. Growth factor-loaded scaffolds for bone engineering. J Control Release 2005;101: 127-136.

34. Lieberman JR, Daluiski A, Stevenson S, Wu L, McAllister P, Lee YP, Kabo JM, Finerman GA, Berk AJ. The effect of regional gene therapy with bone morphogenetic protein-2- 
producing bone-marrow cells on the repair of segmental femoral defects in rats. J Bone Joint Surg Am 1999;81:905917.

35. Wurzler KK, DeWeese TL, Sebald W, Reddi AH. Radiationinduced impairment of bone healing can be overcome by recombinant human bone morphogenetic protein-2. J Craniofac Surg 1998;9:131-137.

36. Chu TM, Warden SJ, Turner CH, Stewart RL. Segmental bone regeneration using a load-bearing biodegradable carrier of bone morphogenetic protein-2. Biomaterials 2007;28:459-467.

37. Niedhart C, Maus U, Redmann E, Schmidt-Rohlfing B, Niethard FU, Siebert $\mathrm{CH}$. Stimulation of bone formation with an in situ setting tricalcium phosphate/rhBMP-2 composite in rats. J Biomed Mater Res A 2003;65:17-23.

38. Ruhe PQ, Hedberg EL, Padron NT, Spauwen PH, Jansen JA, Mikos AG. Biocompatibility and degradation of poly(DL-lac- tic-co-glycolic acid)/calcium phosphate cement composites. J Biomed Mater Res A 2005;74:533-544.

39. Uludag H, D’Augusta D, Golden J, Li J, Timony G, Riedel R, Wozney JM. Implantation of recombinant human bone morphogenetic proteins with biomaterial carriers: A correlation between protein pharmacokinetics and osteoinduction in the rat ectopic model. J Biomed Mater Res 2000;50: 227-238.

40. Uludag H, Gao T, Porter TJ, Friess W, Wozney JM. Delivery systems for BMPs: Factors contributing to protein retention at an application site. J Bone Joint Surg Am A 2001;83 (Suppl):s128-s135.

41. Uludag H, D'Augusta D, Palmer R, Timony G, Wozney J. Characterization of rhBMP-2 pharmacokinetics implanted with biomaterial carriers in the rat ectopic model. J Biomed Mater Res 1999;46:193-202. 